\title{
Opportunistic Unsynchronized Cognitive Radio Networks
}

\author{
Maurice Bellanger \\ Conservatoire National des Arts et Métiers (CNAM), Paris, France \\ Correspondence: bellang@cnam.fr \\ Manuscript communication: received 21 October 2010, accepted 12 January 2011
}

\begin{abstract}
The challenge of opportunistic networks is to maximize the usage of the spectrum by offering quick and easy access to independent systems and their users. A physical layer able to operate efficiently in an unsynchronized environment is needed and it is shown that the filter bank multicarrier (FBMC) technique can meet the objectives in terms of performance and flexibility. Then, a protocol must be developed to ensure global convergence and an overall transmission capacity close to the optimal possible value at a given place and a given time. An approach called "good neighbour" is presented, which consists of building the capacity requested by the users through a threshold regulated local search and with minimum changes in frequency band allocation to reduce interference non-stationarity to other systems. Finally, potential applications of opportunistic networks are reviewed and the conditions for proliferation of this kind of network are discussed.
\end{abstract}

\section{INTRODUCTION}

The radio spectrum has two essential characteristics for communication, it is a limited resource which can be accessed from everywhere. The concept of cognitive radio has been created with the objective to make the best of this situation, by providing the highest spectral efficiency and offering the maximal access flexibility. The ultimate implication of the concept is that a communication system, for example a base station and its users, which detects an unoccupied frequency band in the spectrum should have the freedom to exploit it, without having to go through a lengthy clearance procedure and coordinate with other systems in the same geographical area. Hence, the denomination of opportunistic unsynchronized networks.

In practice, however, the introduction of such new systems amid existing communication networks requires a minimal level of regulation and standardization, in order to ensure coexistence and gain acceptance by all the players in the field. Accordingly, the forthcoming World Radiocommunications Conference in 2012 (WRC'2012) has cognitive radio regulatory policy on its agenda and a question has been put to its technical arm, the International Telecommunication Union-Radio committee (ITU-R) which is preparing a report. In this report, regarding regulation, the emphasis is on the cognitive pilot channel (CPC) and the cognitive control channel (CCC), which exploit a centralized data base to achieve coordinated spectrum sharing and joint radio resource management [1]. The approach is cautious and certainly appropriate, particularly for the CPC when a wide spectrum range is targeted in the entire reachable range from $400 \mathrm{MHz}$ up to $6 \mathrm{GHz}$. However, if the objective is to best exploit a limited band for new services, such as the so-called digital dividend (790-862 MHz and 2500-2690 MHz), the CPC/CCC approach raises a number of issues about the deployment of the corresponding systems, their reaction speed and their real agility, and it is likely to fall short of the ultimate expectations of the cognitive radio concept. In fact, in limited bands, the opportunistic approach is particularly appealing due to its potential agility and the possibility to use a light infrastructure. However, truly opportunistic networks must rely on the capabilities of the terminals, particularly spectrum sensing and monitoring, and some general rules to establish and terminate connections.

The objective of the present paper is to analyze the technical challenges of the opportunistic dynamic spectrum access, propose implementations, review potential applications and discuss conditions for proliferation. The organization is as follows. In Section 2, the constraints imposed by spectrum efficiency and coexistence are discussed and specification objectives are stated. Section 3 is dedicated to the physical layer and the filter bank multicarrier (FBMC) technique is proposed for implementation. The medium access control (MAC) layer is considered in Section 4 and a protocol named "Good Neighbour" is introduced. Potential applications in communication networks are presented in Section 5 and perspectives are discussed in the conclusion.

\section{Technical Challenges}

Two kinds of environments can be considered for the deployment of cognitive radio systems, namely white spaces, or non-occupied frequency bands, in licensed bands and public spaces, which are limited bands reserved for cognitive radio. They lead to the so-called vertical and horizontal spectrum sharing respectively. In both cases, the challenges are in the maximization of the spectral usage under the constraint of coexistence of the users [2].

Coexistence imposes a high level of protection of adjacent users in both the frequency and the time 


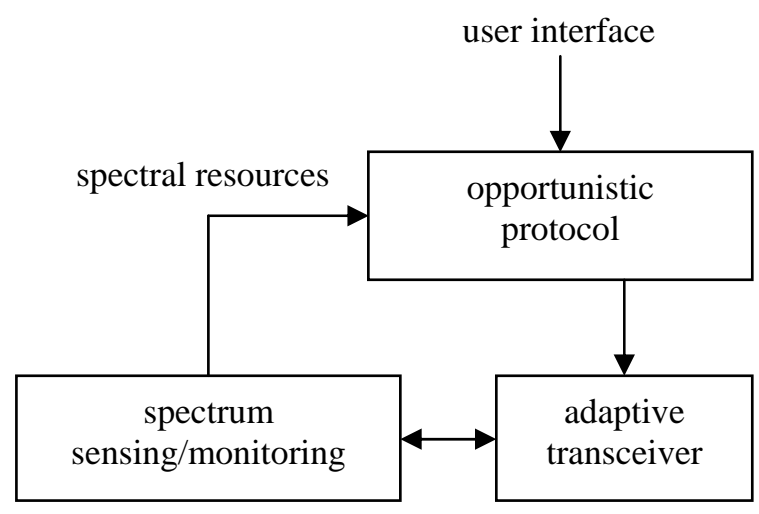

Figure 1. Functionalities of the opportunistic terminal.

domain. Interference attenuation levels have to be specified as well as spectral gaps between users and guard times. The spectral efficiency implies that the time and frequency gaps be minimized. From an opportunistic networking perspective, the decision to access and quit the spectrum is taken by a base station, mainly on the basis of the information provided by the spectrum sensing and monitoring device. Therefore, the performance of this device is critical for the acceptability of the scheme. A block diagram of the terminal is shown in Figure 1. The opportunistic protocol exploits the available spectral resources to adapt the characteristics of the transceiver and meet the needs of the user [3].

In the real time spectrum sensing operation, the analysis is asynchronous because there is no coordination among the sources which produce the received signal. The main parameters are the frequency resolution, the spectral dynamic range (SDR) of the signals to be identified, the accuracy of the noise floor estimations and the latency.

The frequency resolution is determined by the size of the smallest spectral hole which has to be detected. Then, since this bandwidth is to be used for data transmission, the frequency granularity of the transmission system must be considered jointly with the resolution of the sensing device. It is known that maximum transmission efficiency is obtained with multicarrier modulation, where the smallest bandwidth is the subchannel spacing. Therefore, spectrum sensing and multicarrier modulation have the same resolution. In such conditions, it appears natural to use the same device to perform the two functions.

Next, the spectral dynamic range is a crucial parameter for the usefulness of the cognitive radio concept. If secondary (i.e., unlicensed) users are introduced among primary (i.e., licensed) users, it is necessary to take into account the dynamic range of the primary system itself and maintain a high differential power. To give figures, the system should be able to differentiate signals with a power difference of $50 \mathrm{~dB}$ or greater.

Regarding the estimated noise floor, in an unused frequency band, it determines the number of bits of the transmitted symbols. It is the sum of two contributions, the thermal noise and the interference from the active neighbouring bands. The thermal noise cannot be controlled but the measured interference level is determined by the rejection performance of the spectrum analyzer. Rejection figures far beyond the $50 \mathrm{~dB}$ mentioned above are necessary, if high throughput communication is contemplated.

The latency of the sensing device impacts the reaction time of the opportunistic system, which has to be minimized both in accessing and leaving the spectrum. This parameter is tightly related to the sensing techniques employed and the targeted level of performance, for which the following indicators can be considered:ted level of performance, for which the following indicators can be considered:

- detection threshold for the signals of the existing system, i.e., the minimum signal-to-noise ratio (SNR) which is needed,

- detection time for the signals of the existing system, i.e., the duration needed to detect,

- missed detection probability and false alarm probability,

- lost spectrum opportunity ratio, i.e., the expected fraction of the idle time undetected,

- interference ratio, i.e., the expected fraction of the transmission time of the existing system which is interrupted.

A particular aspect of vertical sharing is spectrum monitoring, which is crucial because it delivers the information that triggers the spectrum leaving process when the primary user reappears. Thus, an additional specification is the time it takes to vacate the band. Procedures based on periodical sensing can be elaborated with specified timing, but they increase the latency. A more appropriate approach is continuous monitoring, provided it is compatible with the physical layer.

Once frequency bands have been identified as unoccupied and fit for transmission, the system must decide for the allocation of the available resources to its users. The challenge here lies in the decision process, which must ensure fairness of access to other opportunistic systems, without necessarily coordinating with them. In other words, every system must use only the resources which are necessary to satisfy its clients, in terms of bandwidth and radiated power, and it must facilitate the spectrum access by the other users. In fact, from the perspective of regulatory bodies, the validity of the concept of opportunistic networks will be judged by the global performance in spectrum usage and not by the efficiency of one particular system, and appropriate rules must be elaborated.

\section{Physical Layer for Opportunistic ACCEss}

The objectives of efficiency, opportunistic access and coexistence stated above impose strict constraints on the physical layer. It must provide a high level of spectral separation and have the capability to operate in an unsynchronized environment. The multicarrier technique which is employed in existing systems is OFDM (orthogonal frequency division multiplexing) 
Table I

FREQUENCY COEFFICIENTS FOR PROTOTYPE FILTERS

\begin{tabular}{|c|c|c|c|c|}
\hline$K$ & $H_{0}$ & $H_{1}$ & $H_{2}$ & $H_{3}$ \\
\hline 2 & 1 & 0.707 & - & - \\
\hline 3 & 1 & 0.911438 & 0.411438 & - \\
\hline 4 & 1 & 0.971960 & 0.707 & 0.235147 \\
\hline
\end{tabular}

and it does not satisfy these constraints. An improved physical layer is needed and the FBMC (filter bank multicarrier) technique is proposed as an enhancement of OFDM [4].

Filter banks are efficiently realized by cascading an FFT (fast Fourier transform) and a set of digital filters called a polyphase network (PPN), the PPN being the additional processing that brings high resolution and spectral separation. An introduction to filter banks is given in[4] and a more complete presentation is given in [5]. The number $M$ of filters in the bank is the size of the FFT and the system is said to have $M$ subchannels, while OFDM based on the same FFT has $M$ carriers. The filters are frequency shifted version of the prototype low-pass filter, which is designed according to the Nyquist criterion and using, for example, the classical frequency sampling technique. It is characterized by its length $L$ and the ratio $K=L / M$, called the overlappling factor because it is the number of multicarrier symbols which overlap in the time domain. The frequency response is determined by $K$ samples in the frequency domain, as given by Table 3 for several values of the overlapping factor. Then, the corresponding filter coefficients are given by

$$
\begin{aligned}
& h_{0}=0 ; \\
& h_{i}=1+2 \sum_{k=1}^{K-1}(-1)^{k} H_{k} \cos \left(\frac{2 \pi k i}{K M}\right) ; 1 \leq i \leq K M-1
\end{aligned}
$$

The frequency response of the filter corresponding to the parameters $L=1024, K=4$ and $M=128$ is given in Figure 2, where the unit on the frequency axis is the sub-channel spacing.

It is important to notice that, beyond the neighbouring sub-channels, that is for frequencies greater than 1.5 , the attenuation exceeds $58 \mathrm{~dB}$. In addition, the magnitudes of the ripples decrease monotonically with

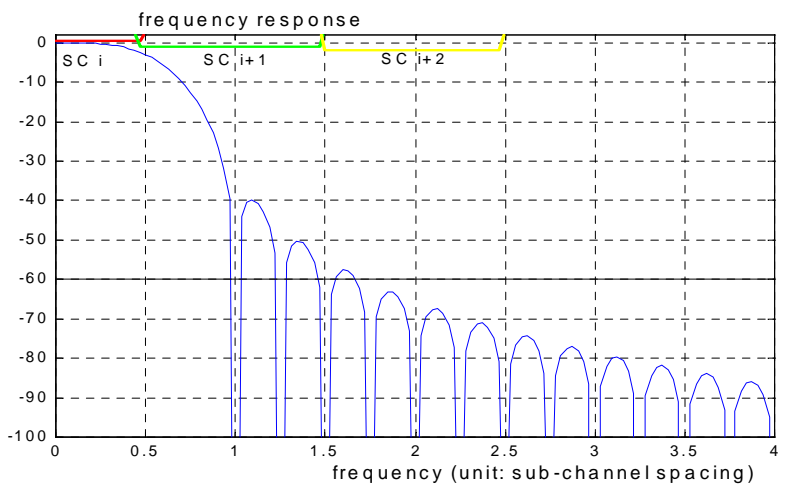

Figure 2. Frequency response of the prototype filter, for a bank of $N=128$ filters.

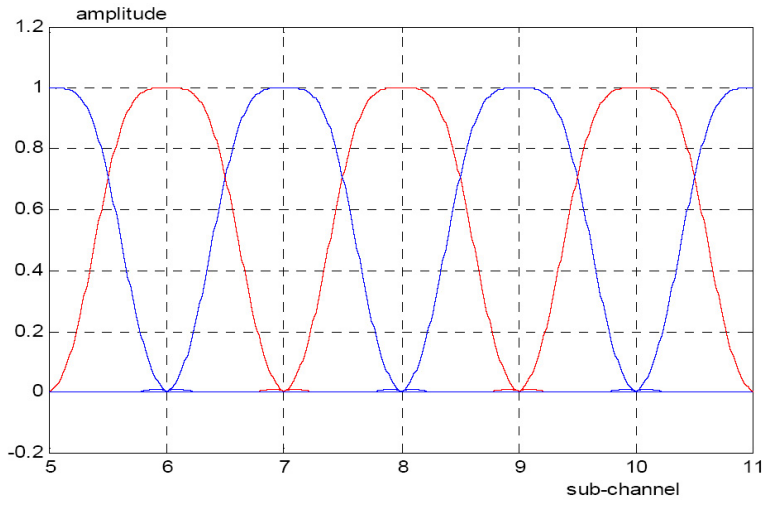

Figure 3. Section of the filter bank for $K=4$.

the frequency which is crucial for interference rejection and noise measurement. The frequency responses of the filters of a section in the bank are shown superimposed in Figure 3. The sub-channels with even index (respectively odd index) do not overlap, which implies that it is sufficient to leave an empty sub-channel between two groups of sub-channels to make them independent. Accordingly, unsynchronized users with different parameters (bandwidth, power, timing, carrier frequency offset) can be accomodated as illustrated in Figure 4. With this kind of filter, the spectral gap between users is one sub-channel width.

As concerns the modulation, the separation of the signals of neighbouring sub-channels is achieved by quadrature, that is using the real input for a given sub-channel and the imaginary input for the neighbours. Then, maximum rate is achieved by the offset quadrature amplitude modulation (OQAM), according to which the rate of the filter bank is doubled and real and imaginary inputs alternate [4-6].

An important feature of the FBMC approach is that the transmission channel impairments can be compensated at the sub-channel level and independently. The block diagram of the receiver is shown in Figure 5. The signal coming from the channel $x(n)$ is applied to the PPN-FFT filter bank which delivers the sub-channel signals. The equalizer includes the compensation of the frequency offset $\delta f$, and a transversal section $H(Z)$ which mitigates the amplitude and phase distortion in the sub-channel. With the OQAM modulation, the receiver filter bank operates at twice the sub-channel frequency spacing, which means that the equalizer is

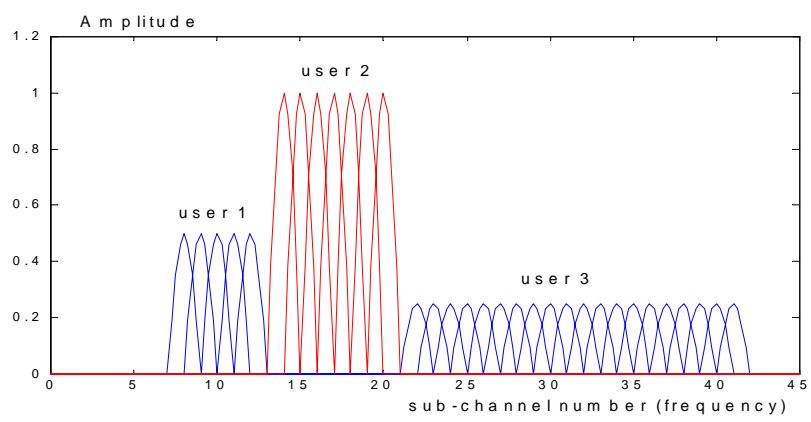

Figure 4. Multicarrier multiuser FBMC transmission. 


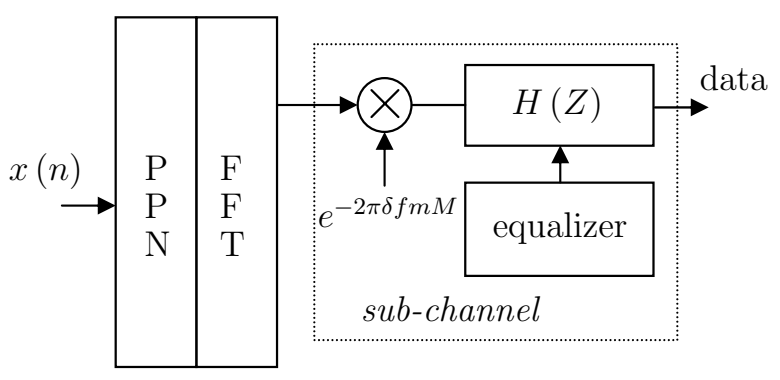

Figure 5. FBMC receiver with equalization.

fractionnaly spaced and, therefore, is able to correct timing offsets. This is crucial for users which are not synchronized, because it means that they can access the system without prior ranging and distant synchronization. As concerns complexity, amplitude and phase distortions require a single tap or a few taps. If significant timing offset is present, more taps are needed. The coefficients are computed from channel measurements through pilot symbols at initialization or scattered pilots during data transmission. Adaptive techniques can also be employed for continuous updating, to get rid of scattered pilots.

Radio networks generally transmit by bursts. With FBMC, the transmission of a burst requires some extra time to accommodate the initial and final transitions associated with the filter impulse response. An illustration is given in Figure 6. In fact, these transitions ensure that the adjacent users are not disturbed when a user accesses or quits the spectrum. If a frequency gap is present between the users or if a temporary reduction in performance is tolerable, the transitions can be significantly shortened, for example to $N_{s}+1$ symbols if $N_{S}$ symbols have to be transmitted. The practical consequence of the increase of the burst length is that FBMC favors longer bursts. In any case, when compared with the OFDM burst, the FBMC burst carries more data symbols in a given time interval, because there is no guard time, or cyclic prefix, between the multicarrier symbols.

The filter bank in the receiver can also be employed for spectrum sensing and the conventional techniques

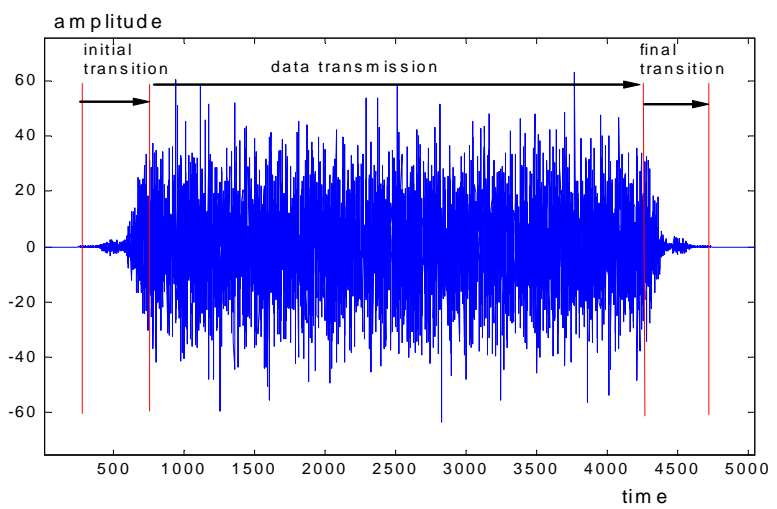

Figure 6. Structure of the transmitted burst with FBMC.

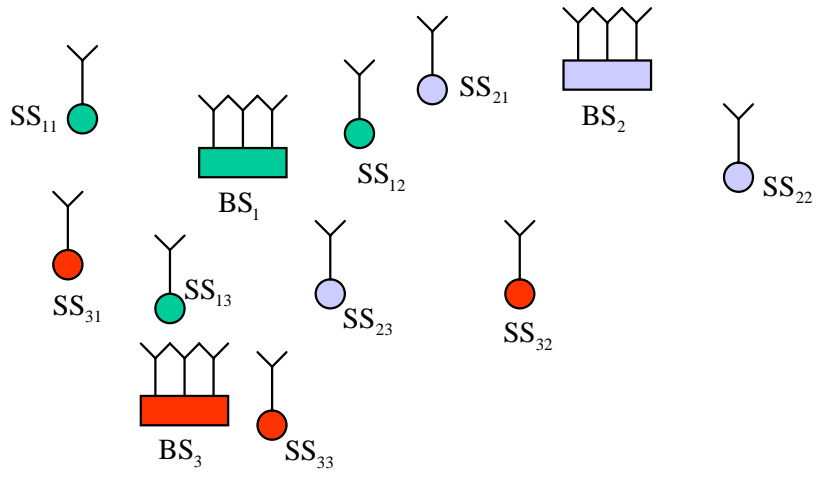

Figure 7. Opportunistic network with 3 base stations.

can be applied at the sub-channel level, namely energy detection, matched filter, cyclostationarity feature detection or self-correlation [7]. The specificity of FBMC lies in the spectral separation provided by the filters and the absence of spectral leakage which improves the performance. In particular, significant gains can be obtained with respect to OFDM concerning noise power estimations. The counterpart is the latency, in which the filter impulse response must be included.

An important aspect is that continuous spectrum monitoring is readily achieved if sub-channels are reserved for this function. In particular, if a group of 3 sub-channels is left idle, the center sub-channel does not overlap with the active sub-channels and it can sense during transmission.

Finally, the conclusion of this section is that a system based on the FBMC physical layer can operate efficiently in an unsynchronized environment. The next step towards building the opportunistic network is the definition of a suitable protocol.

\section{Opportunistic Network}

In some geographical area, independent systems, for example belonging to different providers, operate in a specific frequency range, with the same FBMC physical layer. Each system consists of a base station (BS) and one or several subscriber stations (SS). All the subchannels, or a limited number of them if a primary system is operating in the same frequency range, are accessible and potentially available for every system. The base stations, equipped with single or multiple antennas are sensing the spectrum, in the complete frequency range, to detect the presence of primary users or measure the channel conditions. As an illustration, a network with 3 base stations having 3 subscriber stations each is shown in Figure 6. The base stations dynamically allocate the spectral resources to their users, according to the procedure sketched in Figure 1. If the available capacity, in terms of number of sub-channels and bit rate per sub-channel, is sufficient to meet the needs of the users, the allocation procedure is simple. There is just the option to use more sub-channels than strictly needed and distribute the power or maximize the bit rate and occupy the minimum number of sub- 
channels. The latter approach requires more power, but it facilitates the access for other systems. The specificity of FBMC have to be taken into account while assigning the sub-channels to users, in particular band splitting should preferably be avoided if neighbouring systems or users are not synchronized, because a sub-channel must remain idle at each band edge to ensure independence.

Now, in some circumstances and at some time, the resources might be insufficient and the systems have to compete for the access. Then, some rules must be defined to allow fairness of access, while maximizing the spectrum usage.

A desirable feature is that no system should be prevented from access. Then, some subscribers belonging to different base stations have to share the same subchannel and any new band allocation causes interference to other systems. Note that the multi antenna equipment is critical in that context because it helps mitigate interference. In the sub-channels, the capacity $C$ is determined by the signal-to-interference-plus-noise ratio (SINR) by the expression

$$
C=\Delta f \log _{2}(1+\mathrm{SINR}),
$$

where $\Delta f$ is the sub-channel spacing.

The challenge for the opportunistic protocol is to distribute the total spectral capacity, taking into account the beamforming contribution to interference reduction, among the systems, in real time and with minimum delay. A simple approach, called "good neighbour", has been proposed in [8] and it is based on the following two concepts:

- prevent the extension of the present frequency band and the selection of a new band at some base station for a particular user if its capacity $C$ is already above some threshold $C_{0}$

- minimize the number of band extensions or new band allocations to achieve the given threshold.

The objective of the first concept is to guarantee resource sharing and increase the probability of global convergence of the process. An issue is how to determine the threshold. If it is too low, convergence is fast but some users might be frustrated and the total spectrum capacity might end up below the maximum possible value. If it is too high, convergence may be long or hazardous. Under the hypothesis of noncooperation, the threshold value must be determined locally by each base station. A simple technique consists of exploiting the SINR measurements for all the available sub-channels and taking the average $\mathrm{SINR}_{a v}$. Then a weighting coefficient $\alpha$ is applied to obtain

$$
C=\Delta f \log _{2}\left[1+\alpha \text { SINR }_{a v}\right] .
$$

The coefficient $\alpha$ controls the convergence of the process.

The objective of the second concept is also to speed up convergence by minimizing the nonstationarity caused by the changes in frequency allocations and the resulting interference modifications.

Spectrum sensing is a critical part of the process. If a primary user may be present in the band, the objectives stated in Section 2 apply and spectrum monitoring techniques must be implemented. In case of horizontal sharing also it is necessary to monitor the spectrum in order to detect the changes in the interference environment caused by new allocations of other systems. To achieve this, a base station can introduce sensing intervals between data intervals or distribute pilots in the set of sub-channels it is occupying. As soon as the SINR measurements fall below the threshold, the procedure for acquiring new bands is activated.

Power control is required to reduce the overall interference. It must be pointed out that, due to the separation brought by the filter in FBMC, it can be implemented independently in each base station, provided the number of available sub-channels is sufficient or if the multiantenna receiver is powerful enough to cancel interference. Then, the conventional "water filling" technique applies [9].

Assuming the total power is limited by $Q$, the power $q_{m}$ allocated to user $m,(1 \leq m \leq M)$ having the signalto-noise-plus-interference ratio $\mathrm{SINR}_{m}$ is calculated by

$$
q_{m}=Q \frac{\frac{1}{\mathrm{SINR}_{m}}}{\sum_{i=1}^{M} \frac{1}{\mathrm{SINR}_{i}}} .
$$

Power saving control can be applied to the uplink to increase the battery life of the subscriber stations. It is sufficient to use the power needed to achieve the minimum capacity $C_{0}$.

\section{Applications of Opportunistic NETWORKS}

The introduction of opportunistic networks in some particular frequency bands, such as the digital dividend, will be motivated by their economic impact and the perspective of enhanced spectrum utilization. A number of favorable situations can be envisaged for applications.

High speed local complement to cellular systems: Due to their universality, their rules of deployment and their rigid protocols, cellular systems are unable to support economically the high capacities which might be requested at particular times and particular places by some customers. Opportunistic networks, capitalizing on their capability to adaptively share the spectrum, may have the possibility to build up the frequency bandwidth needed by the high bit rates involved.

Broadband networks in rural areas: Opportunistic networks can help improve the coverage of rural areas and, through dynamic spectrum sharing, offer economical broadband local access to their users. In such a context, they might cooperate with cellular systems or with fixed networks to organize and optimize the global trafic.

Easy and cheap access in sparsely populated areas: With their light infrastructure and their versatility, they can adjust the capacity to the user instantaneous needs and follow the evolution in time and space. 
Temporary high capacity networks for emergency and moving events: At some place and some time, the requested capacity may be so high that the networks are congested. It is the case in emergency situations or for large scale moving events, such as the cycling race "tour de France". Then, opportunistic networks can make the best usage of the instantaneous available spectrum and divert a part of the trafic load.

Peer-to-peer communication: This classical way of communication between users, either people or machines, can be further developed by opportunistic broadband access, in terms of capacity, coverage and mobility.

\section{Conclusion}

The successful large scale introduction of a new radiocommunication paradigm based on spectral sharing will require a number of conditions, in the technical, regulatory and economic domains.

In the technical domain, the compatibility of the opportunistic networks with existing systems must be guaranteed, while preserving spectral efficiency. The relevant parameters must be identified and specifications must be agreed. Among the key parameters are interference levels and signal detection thresholds. In any case, performance is critical.

Regarding regulatory policies, the most important aspect is the spectrum allocation. It can be bands reserved for opportunistic networks or authorized access to already occupied bands under well defined rules and procedures. In this respect, the availability of spectrum space in the UHF band, after the shift from analog to digital television, is a unique opportunity.

An appropriate business model is essential for the start and the sustained growth of opportunistic networks. In fact, the approach will be successful if it is accepted by the administrations and current operators, if it satisfies needs of customers better than existing systems and if it is cost efficient $[10,11]$. Referring to the history of $\mathrm{WiFi}$, the availability of reserved appropriate frequency bands seems essential to launch the process of building the opportunistic business model.

In the end, the operational and commercial success will be materialized by the presence of the opportunistic functionalities in PCs and communication sets.

\section{REFERENCES}

[1] ITU-R, Annex 19 to document 5A/513, "Cognitive radio systems in the land mobile service," International Telecommunication Union (ITU), Tech. Rep., May 31 2010, (working document towards a preliminary draft new report).

[2] L. Berlemann and S. Mangold, Cognitive Radio and Dynamic Spectrum Access. Chichester, UK: John Wiley, 2009.

[3] C.-X. Wang, H.-H. Chen, X. Hong, and M. Guizani, "Cognitive radio network management," IEEE Transactions on Vehicular Technology, vol. 3, no. 1, pp. 28-35, 2008.

[4] M. Bellanger et al., "FBMC physical layer: A primer," PHYDYAS (Physical layer for dynamic access and cognitive radio), Tech. Rep., 2010. [Online]. Available: http://www.ict-phydyas.org
[5] M. Bellanger, Digital Processing of Signals: Theory and Practice, 3rd ed. Chichester, UK: John Wiley, 2000.

[6] B. Hirosaki, "An orthogonally multiplexed QAM system using the discrete Fourier transform," IEEE Transactions on Communications, vol. 29, no. 7, pp. 982-989, 1981.

[7] A. Ghasemi and E. S. Sousa, "Spectrum sensing in cognitive radio networks: Requirements, challenges and design trade-offs," IEEE Communications Magazine, vol. 46, no. 4, pp. 32-39, 2008.

[8] A. M. Kuzminskiy and Y. I. Abramovich, “Decentralized dynamic spectrum allocation based on adaptive antenna array interference mitigation diversity," IEEE Transactions on Signal Processing, vol. 58, no. 4, pp. 2246-2260, 2010.

[9] A. Pascual-Iserte, A. I. Perez-Neira, and M. A. Lagunas, "On power allocation strategies for maximum signal to noise and interference ratio in an OFDM-MIMO system," IEEE Transactions on Wireless Communications, vol. 3, no. 3, pp. 808-820, 2004.

[10] H. Rahul, N. Kushman, D. Katabi, C. Sodini, and F. Edalat, "Learning to share: Narrowband-friendly wideband networks," in Proc. ACM SIGCOMM Conference on Data communication, 2008, pp. 147-158.

[11] P. Bahl, R. Chandra, T. Moscibroda, R. Murty, and M. Welsh, "White space networking with Wi-Fi like connectivity," in Proc. ACM SIGCOMM Conference on Data communication, 2009, pp. 27-38.

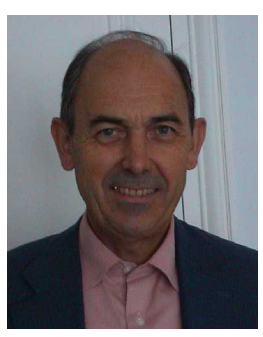

Maurice Bellanger graduated from ENST (Ecole Nationale Supérieure des Télécommunications), Paris, in 1965 and received the doctorate degree from the university of Paris in 1981. He joined the company Philips Communications in France in 1967 and, since then, he has worked on digital signal processing and applications in telecommunications.

In 1991, he joined CNAM (Conservatoire National des Arts et Métiers), a public education and research institute, as a professor of electronics. Since 2008, he is the coordinator of the European Research FP7-project Phydyas.

Elected a fellow of the IEEE in 1984, for contributions to the theory of digital filtering and the applications to communication systems, he was the technical program chairman of the conference ICASSP'82 in Paris. He was the president of EURASIP, the European Association for Signal Processing, from 1986 to 1992 and the chairman of the France section of URSI (Union RadioScientifique Internationale) from 2006 to 2008. He is a member of the French Academy of Technology. 